\title{
New associate Editor Prof. Dr. J. Sehouli for the section Gynecologic Oncology
}

\author{
Jalid Sehouli ${ }^{1}$
}

Published online: 1 December 2016

(C) Springer-Verlag Berlin Heidelberg 2016

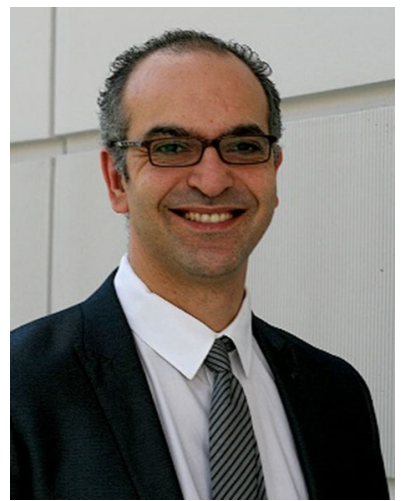

Professor Jalid Sehouli is currently Director of Department of Gynecology, Charité Campus Virchow-Klinikum and

Head of Gynecological Cancer Center Charité in Berlin, Germany. He plays an active role in many key societies and is a Board Member of German Gynaecological Oncology Working Group (AGO) as well as a Chair of the Official Ovarian Cancer Guideline Commission of AGO. $\mathrm{He}$ is also an Official German Member of the Gynaecologic Cancer Intergroup (GCIG) and a founder member, board member and Secretary of the North-Eastern German Society of Gynaecological Oncology. He has published extensively in the area of gynaecological oncology including preclinical, phase I, phase II and phase III trials. His special interest is research on surgical and medical interventions in advanced gynecological malignancies with special focus on translational research and quality of life.

Jalid Sehouli

Jalid.Sehouli@charite.de

1 Department of Gynecology with Center for Oncological Surgery, European Competence Center for Ovarian Cancer Campus Virchow Klinikum and Benjamin Franklin Charité Comprehensive Cancer Center (CCCC), Medical University of Berlin, Augustenburger Platz 1, 13353 Berlin, Germany 